\title{
A Physical Layer Model for G3-PLC Networks Simulation
}

\author{
Alfredo Sanz ${ }^{(1)}$, David Sancho ${ }^{(1)}$, Cristian Guemes ${ }^{(1)}$, José A. Cortés ${ }^{(2)}$ \\ ${ }^{(1)}$ Microchip Technology Inc., Torre C2, Polígono Puerta Norte, A-23, Zaragoza (50821), Spain \\ ${ }^{(2)}$ Universidad de Málaga, Andalucía Tech, Dpto. de Ingeniería de Comunicaciones, \\ E.T.S.I. de Telecomunicación, Campus de Teatinos s/n, Málaga (29071), Spain \\ e-mail: \{alfredo.sanz, david.sancho, cristian.guemes\}@microchip.com, jaca@ic.uma.es
}

\begin{abstract}
This work presents a model of the G3-PLC physical (PHY) layer tailored for network simulations. It allows simulating frequency selective channels with nonstationary colored noise. Collisions with other frames are modeled taking into account the length and the power of the interfering frames. Frame errors are estimated using the effective signal-to-interference-and-noise ratio mapping (ESM) function.

The proposed PHY layer has been integrated into a distributed event-based simulator developed by Microchip. The layer 2+ stack of the simulator uses the same code that actual Microchip G3-PLC devices. Validation has been accomplished by comparing its results to a test network deployed in the laboratory. The latter consists of a coordinator and one hundred meters distributed in 5 levels. Faster-than-real-time simulations and an excellent agreement between the simulated and the measured performance indicators at the application layer have been obtained.
\end{abstract}

\section{INTRODUCTION}

The Smart Grid (SG) area that extends from the medium-voltage to low-voltage (MV/LV) transformers to the customers is referred to as advanced metering infrastructure (AMI). It allows implementing applications like Smart Metering, which is probably the SG area that is currently focusing more deployment efforts [1].

The high data rate narrowband power line communications (NB-PLC) systems standardized by the International Telecommunication Union (ITU) in the Recommendations G.9903 and G.9904 are particularly suited for AMI applications [2]. Indeed, NB-PLC is the most widespread communication technology in the context of Smart Metering in Europe and China [3]. This work concentrates on the G3-PLC system defined in [4].

Network simulators are very useful tools to assess the overall performance of a system, to design the communication stack and to identify the cause of problems that arise in actual deployments. A plethora of simulators are available, e.g. ns-2, ns-3, OMNeT++, JiST/SWANS, GloMoSim. They implement many wired and wireless PHY layer models [5], where the term PHY layer also encompasses the channel model. However, none of them includes a PHY layer of the G3-PLC system.

Designing the PHY layer of a G3-PLC network simulator requires the adoption of channel modeling deci- sions concerning the frequency response (flat/frequency selective), the noise (white/colored), the time variation of the channel and the collisions between frames (limited/full) [5]. Adopting an excessively simplistic model results in faster simulations but, as it happens in the wireless scenario, it may yield to very inaccurate results, in particular when the number of simulated nodes is large [5]. Due to the complexity of the PHY layer processes, a cross-platform implementation where the PHY is implemented in MATLAB and the remaining layers in OMNet++ has been proposed in [6]. However, this approach has difficulty in modeling the collisions adequately.

This paper proposes a PHY layer model for the G3PLC network simulation framework presented in [7]. It allows simulating frequency selective channels with colored noise. The noise profile can be varied along the frame to model the effect of an impulsive noise. The signal-to-interference-and-noise ratio (SINR) resulting from collisions is computed taken into account the frames duration and the channel response between the involved nodes. The implemented PHY is validated by comparing its results with the ones obtained in a test network deployed in the laboratory ${ }^{1}$.

The rest of this paper is organized as follows. Section II summarizes the main characteristics of the G3-PLC PHY layer. Section III briefly describes the structure of the simulator. Section IV details the proposed model for the PHY layer, including the channel model and the procedure employed to estimate the number of errors in the received frames. The validation of the simulator is discussed in section V. Main conclusions are summarized in section VI.

\section{AN OVERVIEW OF THE G3-PLC PHYSICAL LAYER}

The G3-PLC specification covers the PHY, the medium access control (MAC) and the adaptation layers [4]. The MAC layer is based on the IEEE 802.15.4 standard for low-rate wireless personal area network (LoWPAN) [8]. The adaptation layer is based on the

\footnotetext{
${ }^{1}$ Part of these results have been presented at the 10th Workshop on Power Line Communications in Paris, France, 2016.
} 
IPv6 over low-rate wireless personal area network (6LoWPAN) but also includes encryption, a bootstraping mechanism and the layer 2 reactive routing algorithm LOADng [9].

The PHY layer employs a pulse-shaped orthogonal frequency division multiplexing (OFDM) modulation with specific bandplans for the CENELEC-A, FCC and ARIB bands. Table I summarizes the parameters employed in the CENELEC-A and the FCC bands, in which this work is focused. In both cases the discrete Fourier transform (DFT) size is 256 samples, but the maximum number of active carriers is much lower and depends on the bandplan. Some of these tones can be masked to allow coexistence with systems like the one defined in [10]. The set of masked tones is a static personal area network (PAN)-wide parameter. Additionally, when two devices communicate, tones can be adaptively enabled/disabled on a group basis to avoid subbands with low SINR. This is referred to as tone mapping. Coherent and differential phase shift keying (PSK) modulations with 1,2 and 3 bits/symbol are supported in all bandplans.

The employed concatenated forward error correction (FEC) scheme has three working modes: normal, robust and super robust. A Reed-Solomon (RS), a convolutional code and an interleaver are employed in the normal and robust modes. The RS block is 255 bytes but the number of correctable errors, $\mathrm{T}$, depends on the working mode. In the normal mode $\mathrm{T}=8$, while in the robust mode $\mathrm{T}=4$. However, an additional repetition code with rate $1 / 4$ is employed in the latter case. In the super robust mode the FEC consists of the convolutional code, a repetition code with rate $1 / 6$ and the interleaver. The robust and super robust modes can only be employed in conjunction with binary phase shift keying (BPSK) and differential binary phase shift keying (DBPSK).

PHY layer frames consist of a preamble, a frame control header $(\mathrm{FCH})$ and a payload. The preamble and the FCH are transmitted using all available tones (except the masked ones). Tone mapping only applies to the payload. The FCH is always transmitted in the super robust mode using DBPSK in the CENELEC-A band and BPSK in the FCC one. The actual number of OFDM symbols in the FCH depends on the number of masked tones. Table I shows the values that result when the maximum number of carriers is employed.

Payload symbols can be coherently or differentially modulated using the normal or robust mode. When a coherent modulation is employed, two additional symbols denoted as S1 and S2, are inserted between the FCH and the payload for channel estimation and synchronization purposes. In addition, a set of pilot tones are inserted in each payload symbol. The frequency of these tones varies from symbol to symbol.

The payload can carry only one RS block in the
CENELEC-A band and up to two blocks in the FCC one. Hence, the actual number of OFDM symbols depends on the employed modulation and coding scheme. However, they must always be lower than the values indicated in Table I. Moreover, in the CENELEC-A band the number of payload symbols must also be multiple of 4 .

TABLE I

G3-PLC PHY PARAMETERS IN THE CENELEC-A AND FCC BANDS

\begin{tabular}{|c|c|c|}
\hline Parameter & CENELEC-A & FCC \\
\hline Sampling frequency $(\mathrm{kHz})$ & 400 & 1200 \\
\hline $\begin{array}{c}\text { Maximum number of active } \\
\text { carriers }\end{array}$ & 36 & 72 \\
\hline Cyclic prefix (samples) & 30 & 30 \\
\hline First/last carrier index & $23 / 58$ & $33 / 104$ \\
\hline $\begin{array}{l}\text { Pulse-shaped samples at both } \\
\text { symbol ends }\end{array}$ & 8 & 8 \\
\hline Number of preamble symbols & $9+1 / 2$ & $9+1 / 2$ \\
\hline $\begin{array}{l}\text { Default number of FCH } \\
\text { symbols }\end{array}$ & 13 & 12 \\
\hline FCH modulation & $\begin{array}{l}\text { DBPSK super } \\
\text { robust }\end{array}$ & $\begin{array}{l}\text { BPSK super } \\
\text { robust }\end{array}$ \\
\hline $\begin{array}{l}\text { Maximum number of RS } \\
\text { blocks }\end{array}$ & 1 & 2 \\
\hline $\begin{array}{l}\text { Number of carriers in each } \\
\text { tone map group }\end{array}$ & 6 & 3 \\
\hline $\begin{array}{c}\text { Maximum number of payload } \\
\text { symbols }\end{array}$ & 252 & 511 \\
\hline Pilot tones spacing (carriers) & 12 & 12 \\
\hline
\end{tabular}

\section{G3-PLC NETWORK SIMULATOR OVERVIEW}

\section{A. Simulator architecture}

The employed architecture is based on the framework proposed in [7]. Each G3-PLC node (including the coordinator) is simulated by an independent process that implements the full stack, except most parts of the physical layer, and an event machine. Layers are implemented employing the same code used in actual G3-PLC devices by Microchip. A Control module that runs in a different process commands the simulation and ensures its coherence by exchanging events with the event machines of the nodes. The latter run concurrently for a time specified by the Control module. Then they stop and send the frame transmission events to the Network process, which implements the physical layer and the shared power line communications (PLC) channel. It processes events from all the nodes and sends the appropriate events to each of them.

\section{B. Events description}

The Network module processes transmission requests as shown in Fig. 1. It illustrates the events associated to the transmission of two frames in a simplified scenario with three nodes plus the network coordinator. It has been assumed that frames transmitted by node 1 and 
node 3 reach the coordinator and node 2 with a signal level above the receiver sensitivity. Direct communication between node 1 and node 3 is not possible.

Before starting the frame transmission, node 1 and node 3 send a TX_REQUEST event (not shown in Fig. 1) to the Network module. Since the channel is idle at the senders location, the Network module sends a TX_START event to each sender, indicating that its frame is being transmitted, and notifies all the nodes that receive these frames with a signal level above the receiver sensitivity that a frame preamble has been detected (CARRIER_DET). If the senders were already receiving a frame (even if it were destined to another node), the Network process would have answered that the channel were busy and transmissions would not have been performed. In the situation shown in Fig. 1 , the Network module notifies each sender that its frame has been transmitted (TX_END). Then, it informs the destiny that reception is completed and reports the number of errors in the received frame (FRAME_RX). It is interesting to note that the coordinator has not received a FRAME_RX signal from the Network module corresponding to the frame with $\mathrm{ID}=2$. The reason is that the coordinator was already synchronized to frame with $\mathrm{ID}=1$. Hence, frame with $\mathrm{ID}=2$ is seen as noise. To conclude, the Network module communicates to the remaining nodes that were receiving the frame that the channel is now idle (CHANNEL_IDLE).

\section{PHY LAYER MODELING}

The metric used to abstract the PHY layer to the upper ones is the number of errors in the received frames. To this end, the SINR at the receiver is firstly computed. This magnitude may be colored and time-variant. Then, the frame error rate (FER) corresponding to each SINR region is estimated using the ESM function proposed in [11]. This function has to be parameterized for the transmission modes used in G3-PLC. To this end, an accurate characterization of the FER in the additive white Gaussian noise (AWGN) channel is required.

This section describes the three aforementioned elements: the channel model used as the basis for the SINR calculation, the characterization of the PHY layer in the AWGN channel and the procedure employed to parameterize the ESM function.

\section{A. Channel model}

Let us consider a desired frame with $M$ OFDM symbols transmitted from node $s$ to node $r$ using the set of carriers $\mathcal{K}=\left\{k_{0}, \ldots, k_{|\mathcal{K}|-1}\right\}$. Node $r$ receives the constellation value transmitted in the $k$ th carrier of the $m$ th symbol of this frame with a SINR given by

$$
\gamma_{s, r}(k, m)=\frac{P_{s}(k) \alpha_{s, r}(k)}{N_{r}(k)+\sum_{i \in \mathcal{I}} A_{i}(m) P_{i}(k) \alpha_{i, r}(k)},
$$

with $k \in \mathcal{K}, m=\{0, \ldots, M-1\}$, and where $P_{s}(k)$ is the power allocated by node $s$ to carrier $k, \alpha_{s, r}(k)$ is the attenuation of the channel between nodes $s$ and $r$ in carrier $k$, and $N_{r}(k)$ is the noise power that impairs the $k$ th carrier at node $r$. The summation in the denominator of (1) models the effect of the collisions with frames transmitted by the set of interfering nodes $\mathcal{I}$, which are assumed to be independent and uncorrelated between them and with the noise. Hence, $A_{i}(m)=1$ if node $i$ is transmitting a frame while node $r$ is receiving the $m$ th symbol of the desired frame. Otherwise, $A_{i}(m)=0$.

Expression (1) implements the so-called full interference model [5]. It allows simulating frequency selective channels with colored noise. Moreover, the value of $N_{r}(k)$ used in each frame is drawn from a random variable (RV) with configurable mean and variance, which can be used to model the effect of impulsive noise.

\section{B. FER in AWGN}

The FER values in the AWGN channel needed to parameterize the ESM function have been obtained by means of simulations performed in MATLAB. Both the sampling frequency offset (SFO) and the channel estimation errors have been taken into account. It is interesting to notice that the latter errors cause the actual SINR at the demodulator output, $\gamma(k)$, to be no longer white. Fig. 2 (a) and (b) illustrate this end. They depict the relation between $\gamma(k)$ and the channel signal-tonoise ratio (SNR), $\gamma_{\mathrm{AWGN}}$, for each carrier of the FCC band when the payload is modulated using DBPSK and BPSK, respectively. Curves corresponding to the first and the last carriers have been highlighted in red and black, respectively. The SFO has been fixed to the maximum value allowed in the G3 specification, i.e., $50 \mathrm{ppm}$. The displayed SNR range corresponds to FER values in the range $10^{-3} \leq \mathrm{FER}<1$.

Fig. 2 (a) shows that high frequency carriers experience lower SINR values than low frequency carriers when DBPSK is used. This is due to the SFO estimation error, whose effect on the received constellations can be approximated by a phase error that is proportional to the carrier index. The SINR difference between carriers increases as $\gamma_{\mathrm{AWGN}}$ increases because the SFO estimation errors dominate over the channel noise. This is in contrast to the BPSK case displayed in Fig. 2 (b), where the SINR difference between carriers decreases as the $\gamma_{\mathrm{AWGN}}$ increases. The reason is that the SFO and the channel estimations are refined in the payload using the pilot tones. The improvement given by this refinement increases as the channel noise decreases. It is also interesting to note that there is a slight SINR difference between adjacent carriers. This is also due to the channel estimation refinement performed using the pilot tones. The frequency of the latter vary from symbol to symbol. However, they are only transmitted 

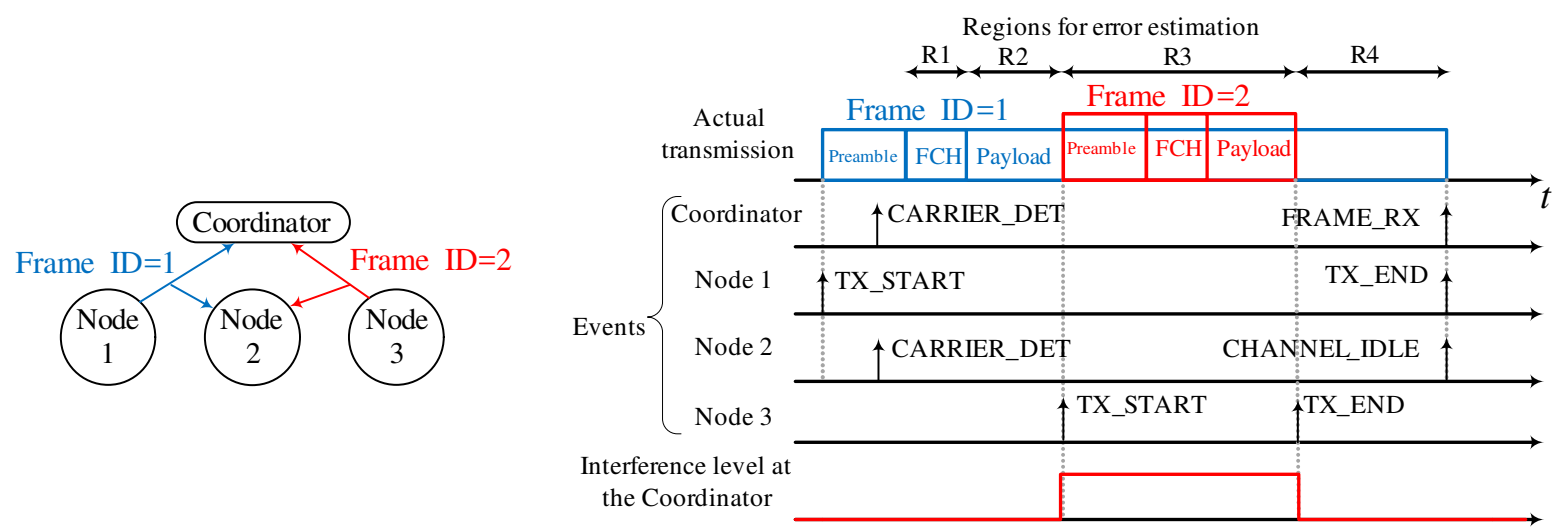

Fig. 1. Simplified transmission example and corresponding events and regions used for errors estimation.

in carriers with even indexes, except for the last one, which is not used as pilot despite it has an even index. As a result, channel estimation is worse in carriers that are never used as pilots.

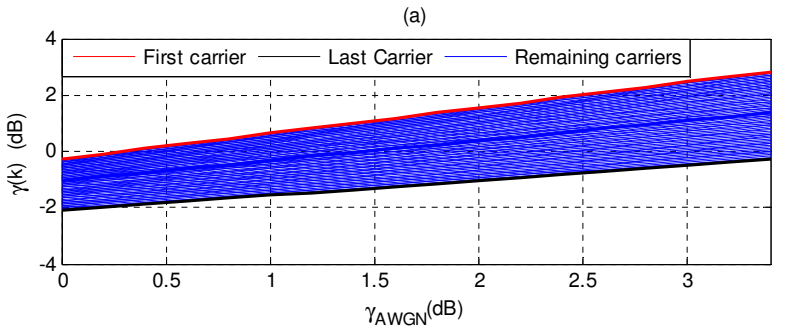

(b)

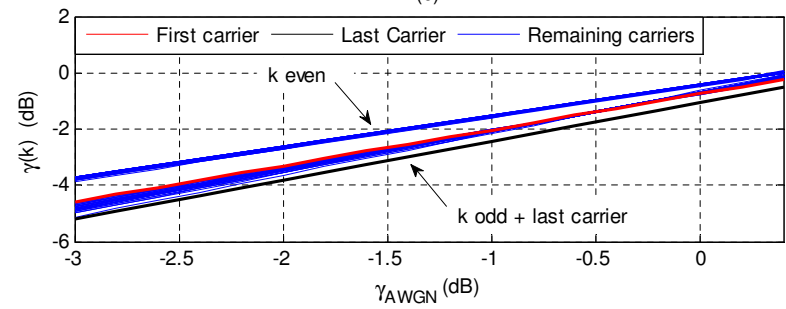

Fig. 2. Relation between the SNR of the AWGN channel and the actual SINR at the demodulator output for: (a) DBPSK and (b) BPSK.

Fig. 3 displays the FER values obtained in the aforementioned conditions. $\gamma_{\mathrm{AWGN}}$ denotes the channel SNR. One RS block is considered in both the CENELEC$A$ and FCC bands. As seen, FER values have been computed separately for the FCH and the payload. In the former case, only errors in the $\mathrm{FCH}$ are taken into account, while in the latter only errors in the payload are considered. It is interesting to note that, in the CENELEC-A band, the FER of the payload when using robust BPSK is much better than the FER of the FCH. This indicates that the actual performance when using robust BPSK will be about $2 \mathrm{~dB}$ lower than expected from the FER of the payload. This is due to the higher FER of the differential modulation used in the $\mathrm{FCH}$ with respect to the coherent one used in the payload. In the FCC band, the FER of the FCH in the region $\operatorname{FER}_{\text {AWGN }}\left(\gamma_{\text {AWGN }}\right)<10^{-2}$ is slightly worse than the one of the payload with robust BPSK. This is due to the larger error of the SFO estimate used to demodulate the $\mathrm{FCH}$, which is obtained exclusively from the preamble symbols, with respect to the SFO estimate employed to demodulate the payload, which is estimated from the preamble, the FCH, the S1-S2 symbols and the pilots.

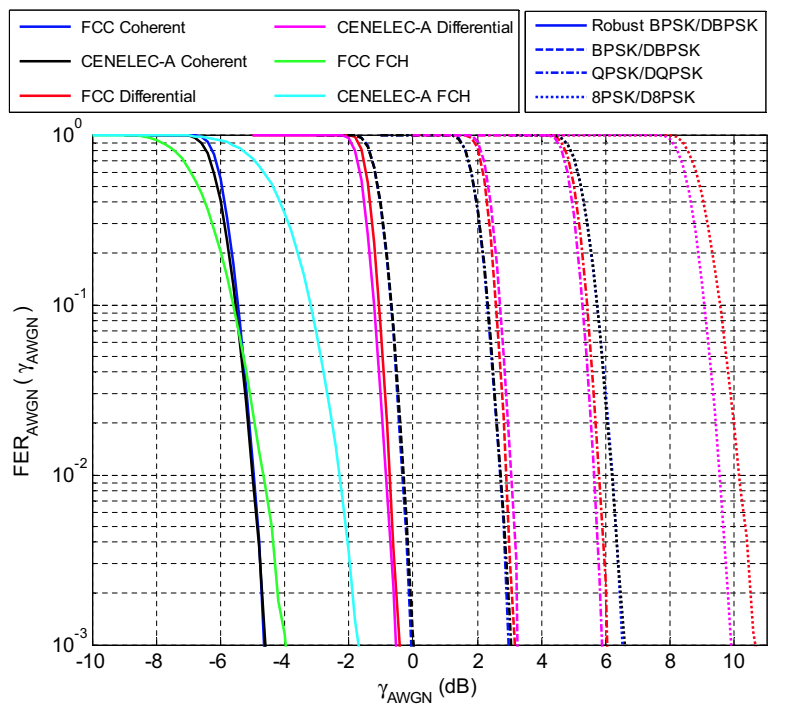

Fig. 3. Estimated FER in AWGN channel corresponding to the FCH and the payload in the CENELEC-A and FCC bands.

FER values shown in Fig. 3 have been computed using all the available tones and the largest payload allowed in each transmission mode. The FER decreases as the payload length is reduced because the RS code has a fixed correction capacity. However, accomplished simulations indicate that performance improvements for shorter (but realistic) payload lengths are below $1 \mathrm{~dB}$ for FER values in the range $10^{-2} \leq \mathrm{FER}<1$.

The FER is also affected by the number and fre- 
quency index of the masked carriers. As mentioned, an uncorrected SFO results is a symbol phase error that is approximately proportional to the carrier index. Hence, the SFO estimation is more affected by the masking of high frequency carriers than by the masking of low frequency ones. Nevertheless, FER degradation due to practical tome masking is negligible when the payload is differentially modulated. When the payload is coherently modulated, the largest degradation occurs in the robust mode, where the strong coding scheme obliges the SFO estimator to work in a very low SNR regime. However, simulations indicate that the degradation is below $1 \mathrm{~dB}$ for FER values in the range $10^{-2} \leq \mathrm{FER}<1$, even when the lower half of the carriers are masked.

\section{FER estimation in time and frequency-selective channels}

Frames are divided into regions consisting of an integer number of OFDM symbols with the same transmission mode and in which $\gamma_{s, r}(k, m)=\gamma(k)$. The example in Fig. 1 defines 4 regions for the estimation of the errors in the frame with $\mathrm{ID}=1$. The first region comprises the FCH. During the second region, the payload is being received and no interfering frames exist. Since the FCH and the payload employ different coding schemes, at least two regions are needed in all frames. The third region is defined to take into account the effect of the interfering frame with $\mathrm{ID}=2$. The fourth region begins just after the interference disappears and lasts until the frame ends.

The number of bit errors in each region is estimated from the FER, which is computed using the ESM function proposed in [11]. According to it, the FER of an OFDM system whose carriers experience a colored SINR given by $\{\gamma(k)\}$, with $k \in \mathcal{K}$, is given by

$$
\operatorname{FER}(\{\gamma(k)\})=\operatorname{FER}_{\mathrm{AWGN}}\left(\gamma_{e}\right),
$$

where $\operatorname{FER}_{\text {AWGN }}\left(\gamma_{e}\right)$ is the FER achieved by the same OFDM system in an AWGN channel with SNR equal to

$$
\gamma_{e}=-\beta \log \left(\frac{1}{|\mathcal{K}|} \sum_{k \in \mathcal{K}} e^{-\frac{\gamma(k)}{\beta}}\right),
$$

where $\gamma_{e}$ is referred to as the effective SINR and $\beta$ is a parameter that depends on the modulation and the coding scheme, but not on the channel characteristics.

Following a similar approach to the one in [12], [13], the optimum value of $\beta$ has been obtained as the solution to the least-squares minimization problem,

$$
\beta^{*}=\arg \min _{\beta}\left\{\sum_{j \in \mathcal{J}}\left|\gamma_{e}^{j}(\beta)-\gamma_{\mathrm{AWGN}}^{j}\right|^{2}\right\},
$$

where $\gamma_{e}^{j}(\beta)$ is the effective SNR corresponding to a channel state $j$, characterized by $\left\{\gamma^{j}(k)\right\}$, and $\gamma_{\text {AWGN }}^{j}$ is such that $\operatorname{FER}\left(\left\{\gamma^{j}(k)\right\}\right)=\operatorname{FER}_{\text {AWGN }}\left(\gamma_{\text {AWGN }}^{j}\right)$.

The $\operatorname{FER}_{\mathrm{AWGN}}\left(\gamma_{\mathrm{AWGN}}\right)$ values used in the optimization process are the ones shown in Fig. 3. Similarly, the values of $\left\{\gamma^{j}(k)\right\}$ and $\operatorname{FER}\left(\left\{\gamma^{j}(k)\right\}\right)$ used in (4) have been obtained by means of simulations in colored Gaussian noise with thousands of channel states covering the range $10^{-3} \leq \operatorname{FER}\left(\left\{\gamma^{j}(k)\right\}\right)<1$. Table II shows the optimum values of $\beta$ for the transmission modes used in the payload.

TABLE II

VALUES OF $\beta^{*}$ USED IN THE ESM FUNCTION IN THE CENELEC-A AND FCC BANDS

\begin{tabular}{ccc} 
Modulation & CENELEC-A & FCC \\
\hline Robust BPSK & 0.2 & 0.2 \\
BPSK & 0.4 & 0.3 \\
QPSK & 0.7 & 0.8 \\
8PSK & 1.5 & 1.6 \\
Robust DBPSK & 1.4 & 1.3 \\
DBPSK & 1.1 & 1.0 \\
DQPSK & 1.6 & 1.8 \\
D8PSK & 4.2 & 4.7 \\
\hline
\end{tabular}

\section{VALIDATION AND NETWORK PERFORMANCE ANALYSIS}

Two procedures have been employed to validate the proposed PHY layer model. The first one assesses the goodness of the ESM approach to estimate the FER in point-to-point links where the channel response is frequency selective and the noise is colored and stationary. Neither noise level variations nor collisions occur. Hence, the PHY layer can be tested as a stand-alone process. The second procedure is intended to validate the integration of the PHY with the remaining layers of the network simulator and its capacity to estimate frame errors in a shared medium where collisions may occur.

Regarding the first procedure, Fig. 4 displays the cumulative distribution function (CDF) of the error between $\gamma_{e}^{j}\left(\beta^{*}\right)$ and $\gamma_{\mathrm{AWGN}}^{j}$, with $j \in \mathcal{J}$, when using the values of $\beta^{*}$ given in Table II. As seen, the absolute value of the error is below $1 \mathrm{~dB}$ in $96 \%$ of the cases and lower than $0.5 \mathrm{~dB}$ in $80 \%$ of the cases, except for BPSK in the FCC band, where this percentage is a bit lower, but still higher than $70 \%$.

In the second procedure, simulated results have been compared to the ones obtained in a test network deployed in the laboratory. The latter consists of the coordinator and 100 nodes distributed in 5 levels. A flat attenuation of $50 \mathrm{~dB}$ is introduced between each level and also between the coordinator and the first level. A line impedance stabilization network (LISN) is used to control the noise level in the network, which is fixed to 


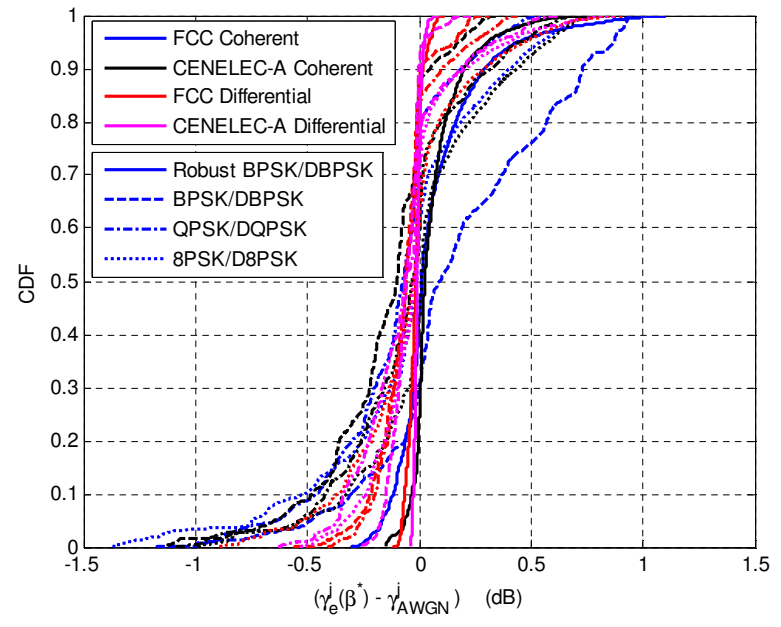

Fig. 4. CDF of the error between the values of $\gamma_{e}^{j}\left(\beta^{*}\right)$ and $\gamma_{\mathrm{AWGN}}^{j}$, with $j \in \mathcal{J}$, for FER values in the payload.

ensure that coherent and differential 8PSK modulations can be employed. By doing so, the capacity to simulate the adaptive modulation process defined the G3-PLC specification can be also tested. The application layer emulates the DLMS/COSEM protocol, which is the one used in Smart Metering.

Simulation has been executed in a Linux operative system that runs in a Dell Precision T7600 workstation equipped with two Intel Xeon CPUs (E5-2687W) at 3.10 $\mathrm{GHz}, 32$ Gbytes of RAM and two SAS hard drives. The simulated-time to real-time ratio for the tested network is $17 / 60$, i.e. faster than real-time. Table III shows the bootstraping time (time for all the nodes to get registered), the average cycle time (time to read the energy consumption of all the nodes) and the maximum number of hops (number of times that a frame is relayed). As seen, there is an excellent matching between the measured and the simulated results.

TABLE III

SIMULATED AND MEASURED PERFORMANCE VALUES

\begin{tabular}{ccc} 
Performance indicator & Simulated & Measured \\
\hline Bootstrap time (minutes) & 53 & 52 \\
Average cycle time (s) & 680 & 680 \\
Maximum number of hops & 5 & 5 \\
\hline
\end{tabular}

\section{CONCLUSION}

This work has presented a PHY layer model of the G3-PLC system specifically designed for network simulations. It implements a frequency and time-variant channel model in which collisions are modeled using a full interference approach. The metric used to abstract the PHY layer is the number of errors in the received frames, which are estimated from the SINR using the ESM function.
The presented PHY has been embedded into a network simulator developed by Microchip. It has been verified that in a point-to-point link with a frequency selective channel and stationary colored noise, errors in the estimated FER are lower than $1 \mathrm{~dB}$ in $96 \%$ of the cases and lower than $0.5 \mathrm{~dB}$ in almost $80 \%$ of the cases.

Its performance in a realistic network has been validated by comparing the simulated results results to the ones obtained in a test network with one hundred meters deployed in the laboratory. The simulated performance indicators at the application layer excellently match the measured ones and the simulation time is about 3.5 times lower than the real time.

\section{ACKNOWLEDGMENT}

The authors thank Microchip Technology Inc. for the support provided to accomplish this work.

\section{REFERENCES}

[1] A. Sendin, I. Peña, and P. Angueira, "Strategies for power line communications smart metering network deployment," Energies, vol. 2014, no. 7, pp. 2377-2420, 2014.

[2] S. Galli, A. Scaglione, and Z. Wang, "For the grid and through the grid: The role of power line communications in the Smart Grid," Proceedings of the IEEE, vol. 99, no. 6, pp. 998-1027, June 2011.

[3] N. Uribe-Pérez, L. Hernández, D. de la Vega, and I. Angulo, "State of the Art and Trends Review of Smart Metering in Electricity Grids," Applied Sciences, vol. 6, no. 3, pp. 1-24, 2016.

[4] "Narrowband orthogonal frequency division multiplexing power line communication transceivers for G3-PLC networks," ITU-T Recommendation G.9903, March 2013.

[5] E. Ben Hamida, G. Chelius, and J.-M. Gorce, "Impact of the Physical Layer Modeling on the Accuracy and Scalability of Wireless Network Simulation," SIMULATION (Transactions of the Society for Modeling and Simulation International), vol. 85, no. 9, pp. 574-588, September 2009.

[6] L. Di Bert, S. D“Alessandro, and A. M. Tonello, "A G3-PLC Simulator for Access Networks," in Proceedings of the IEEE International Symposium on Power Line Communications and its Applications, March 2014, pp. 99-104.

[7] A. Sanz, P. J. Piñero, S. Miguel, and J. I. García-Nicolás, "Distributed Event-Driven Simulation Environment for Smart Metering Protocols Evaluation," in Proceedings of the IEEE International Conference on Smart Grid Communications, 2012, pp. $151-156$.

[8] "Part 15.4: Wireless medium access control (mac) and physical layer (phy) specifications for low-rate wireless personal area networks (wpans)," IEEE Std 802.15.4-2006, 2006.

[9] T. Clausen et al., "The Lightweight On-demand Ad hoc Distance-vector Routing Protocol Next Generation (LOADng)," IETF Internet-Draft, 2016.

[10] IEC 61334-5-1, "Distribution automation using distribution line carrier systems Part 5-1: Lower layer profiles-The spread frequency shift keying (S-FSK) profile," 2001.

[11] Ericsson, "System-level evaluation of OFDM - further considerations," 3GPP TSG-RAN WG1 35, R1-031303, Tech. Rep., 2003.

[12] E. Tuomaala and H. Wan, "Effective SINR approach of link to system mapping in OFDM/Multi-carrier mobile network," in Proceedings of the International Conference on Mobile Technology, Applications and Systems, November 2005.

[13] J. A. Cortés, A. Sanz, P. Estopiñán, and J. I. García, "Performance Assessment of OFDM-based Narrowband PLC for Advanced Metering Infrastructure," in Proceedings of the IEEE International Symposium on Power Line Communications and its Applications, 2016, pp. 132-137. 